\title{
Estado nutricional de gestantes avaliado por três diferentes métodos de classificação antropométrica
}

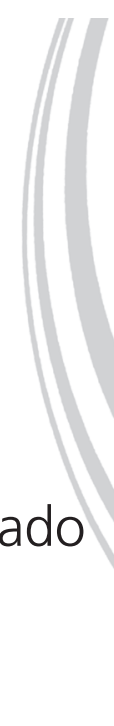

\author{
Nutritional status of pregnant women according \\ to three different anthropometric \\ classification methods
}

\author{
Maria Inês Bezerra de MELO' \\ Ariani Impieri de SOUZA' \\ José Natal FIGUEIROA' \\ José Eulálio CABRAL-FILHO' \\ Maria Helena D'Aquino BENÍCIO' \\ Malaquias BATISTA-FILHO'
}

RE S U M O

\section{Objetivo}

Avaliar o estado nutricional de gestantes por três diferentes métodos.

\section{Métodos}

Estudo de corte transversal, realizado entre janeiro e junho de 2006, com 992 gestantes de um serviço público. O estado nutricional foi classificado por diferentes métodos de avaliação: nomograma de Rosso, método de Atalah e gestograma do Centro Latino Americano de Perinatologia. Os resultados foram comparados com o estado nutricional de mulheres não gestantes em idade reprodutiva da Região Nordeste e do Brasil. As diferenças foram analisadas pelo teste qui-quadrado de bondade de ajuste, adotando-se o nível de significância de $5 \%$.

\section{Resultados}

O baixo peso gravídico avaliado pelo nomograma de Rosso foi a condição que mais contribuiu para a diferença encontrada $(p<0,001)$. Pelo método de Atalah, as diferenças extremas também foram observadas nas prevalências de baixo peso gravídico tanto no grupo de mulheres da Região Nordeste quanto do Brasil. Em relação ao gestograma do Centro Latino Americano de Perinatologia, os maiores diferenciais de prevalência também ocorreram na condição de baixo peso: $18 \%$ versus 7,4\% para a Região Nordeste e 18\% versus 6,7\% para o Brasil, embora tenha sido o método de avaliação que mais se aproximou dos resultados da população de comparação.

\footnotetext{
${ }^{1}$ Instituto de Medicina Integral Prof. Fernando Figueira, Departamento de Pesquisas. R. dos Coelhos, 300, Boa Vista, 50070-550, Recife, PE, Brasil. Correspondência para/Correspondence to: A.I. SOUZA. E-mails: <ariani@imip.org.br>; $<$ arianii@terra.com.br>.
} 
586 | M.I.B. MELO et al.

\section{Conclusão}

A distribuição do estado nutricional, avaliado pelos três métodos, foi diferente da distribuição nutricional da população de não gestantes tanto em nível regional quanto em nível nacional, particularmente o Nomograma de Rosso, demonstrando utilidade limitada desses instrumentos. A avaliação pelo Centro Latino Americano de Perinatologia é a que menos se distancia da situação encontrada para não gestantes.

Termos de indexação: Antropometria. Avaliação. Estado nutricional. Gestação.

\section{A B S T R A C T}

\section{Objective}

This study assessed the nutritional status of pregnant women by three different methods.

\section{Methods}

A cross sectional study was conducted between January and June 2006 with 992 pregnant women attending a public prenatal care facility. Nutritional status was classified according to different assessment methods (Rosso nomogram, Atalah method and the Latin American Center for Perinatology gestogram). The results were compared with the nutritional status of nonpregnant women in childbearing age in the Brazilian Northeast and all of Brazil. The differences were analyzed by the chi-square goodness of fit test, with a significance level of $5 \%$.

\section{Results}

Low weight of pregnant women according to the Rosso nomogram was the condition that most contributed to the difference $(p<0.001)$. According to the Atalah method, extreme differences were also observed in the prevalences of underweight pregnant women both in the Northeast and in all of Brazil. In relation to the gestogram of the Perinatology Latin American Center, the greatest prevalence differences also occurred among the underweight: $18 \%$ versus $7.4 \%$ for the Northeast Region and $18 \%$ versus $6.7 \%$ for Brazil, although this was the assessment method that came closest to the results of the population being compared.

\section{Conclusion}

Nutritional status distribution of pregnant women, assessed by three methods, was different from the nutritional status distribution of the nonpregnant population, both regionally and domestically, especially according to Rosso's nomogram, demonstrating the limited use of these instruments. Assessment by the Perinatology Latin American Center comes closest to the nutritional status distribution of nonpregnant women.

Indexing terms: Anthropometry. Evaluation. Nutritional status. Pregnancy.

\section{N T R O D U Ç Ã O}

Os desvios do estudo nutricional - sejam eles devidos a carências globais ou específicas, sejam por excessos como a obesidade - representam agravos de grande importância para a saúde individual e coletiva ${ }^{1}$. Nessa perspectiva, o período gestacional configura uma situação de marcante vulnerabilidade, principalmente diante de condições socioeconômicas desfavoráveis² ${ }^{2}$ No Brasil, notoriamente pela rápida instalação da transição nutricional ${ }^{3}$, esses aspectos assumem uma pertinência peculiar, levando-se em conta as estatísticas preocupantes de morbimortalidade materna ${ }^{4,5}$. Chamam a atenção as grandes discre- pâncias observadas no Brasil nas avaliações antropométricas do estado nutricional de mulheres não gestantes em relação às gestantes ${ }^{6}$, agravadas ainda pelas diferenças encontradas na própria avaliação das mulheres grávidas entre si, em função dos diferentes métodos aplicados em sua classificação antropométrica ${ }^{7-13}$.

O Brasil pode ser considerado um exemplo dos problemas metodológicos referentes à avaliação antropométrica do estado nutricional de gestantes. Assim, nos últimos 25 anos, três diferentes modelos têm sido utilizados nos serviços pré-natais por recomendação do Ministério da Saúde do Brasil: o nomograma de Rosso ${ }^{14}$, a curva de Atalah ${ }^{15}$ e o gestograma proposto pelo Centro 
Latinoamericano de Perinatologia (CLAP), experimentalmente aplicado em alguns estados a partir do ano 2000, tendo como princípios normativos o estudo de Fescina, divulgado pela Organização Panamericana de Saúde (OPAS), em $1983^{10}$. Tais métodos de avaliação antropométrica utilizados dentro e fora do Brasil não têm, até agora, apresentado resultados plenamente convincentes, seja em termos conceituais, seja por conta dos desfechos considerados para sua validação, implicando várias discordâncias' ${ }^{16,17}$.

Assim, à medida que os resultados tornam-se marcantemente conflitantes, evidencia-se a pertinência de estudos de validação dos métodos utilizados como forma de compreender suas possíveis limitações e relativizar sua interpretação.

Neste estudo, objetiva-se a análise dos resultados por um critério epidemiológico peculiar: as discordâncias e as concordâncias comparativas com valores esperados a partir do perfil apresentado pela população de mulheres não gestantes, em idade reprodutiva, em nível regional (Nordeste) e nacional (Brasil). Tais valores foram utilizados como referência para avaliar uma população de gestantes assistidas em um serviço de saúde credenciado como referência para a área materno-infantil pelo Ministério da Saúde

\section{MÉ T O D O S}

Foi realizado um estudo observacional, do tipo corte transversal, entre janeiro e junho de 2006, com 992 gestantes assistidas no pré-natal de uma maternidade pública na cidade do Recife (PE). As gestantes foram selecionadas consecutivamente, à medida que compareciam ao ambulatório para a realização da consulta pré-natal. Foram excluídas da pesquisa todas as gestantes que apresentaram alguma doença associada de relevância clínica, como os casos de má formação física congênita ou adquirida, que pudesse interferir na aferição da antropometria. Foram excluídas também as gestantes com idade inferior a 18 anos.
O estado nutricional foi classificado por diferentes métodos de avaliação (nomograma de Rosso, método de Atalah e gestograma do (LAP), durante o segundo ou terceiro trimestres gestacionais. Como referência de validação, os resultados da aplicação de cada método de avaliação antropométrica foram comparados com os valores que seriam esperados a partir do estado nutricional de mulheres não gestantes em idade reprodutiva da Região Nordeste e do Brasil ${ }^{18}$. Desse modo, tratou-se de um critério epidemiológico de validação, admitindo-se o consenso em torno do Índice de Massa Corporal (IMC) considerado padrão-ouro como método de avaliação de muIheres adultas no período reprodutivo e o dissenso entre os métodos usuais de classificação ponderal de gestantes. Para o cálculo dos valores teoricamente esperados, foi aplicada a distribuição de resultados da amostra em três categorias (baixo peso, peso normal e sobrepeso/obesidade) às ponderações ou ajustes dos percentuais derivados de avaliações representativas da população de mulheres em idade reprodutiva no Nordeste e no Brasil no ano de $2005^{18}$. Esses percentuais foram descritos na coluna "valores esperados" de cada tabela.

As diferenças para mais (sobrepeso/obesidade) ou para menos (baixo peso), situações de interesse clínico e epidemiológico, foram analisadas pelo teste qui-quadrado de bondade de ajuste, adotando-se o nível de significância de 5\% para risco de rejeição da hipótese nula. Na comparação dos diferentes métodos, foi analisada a contribuição de cada discrepância em relação ao valor total do qui-quadrado, tomando-se como referência os valores esperados por critérios epidemiológicos, ou seja, as frequencias esperadas a partir das classificações de IMC de mulheres do Nordeste do país e do Brasil como um todo.

O estudo cumpriu os princípios éticos da Declaração de Helsinki (2000), bem como da Resolução 196/96 do Conselho Nacional de Saúde do Brasil, e foi aprovado pelo Comitê de Ética e Pesquisa em Seres Humanos do hospital onde o estudo foi desenvolvido e registrado sob o número $726 / 2006$. 


\section{RE S U L T A D O S}

Em relação à caracterização da população, mais da metade das 992 gestantes estudadas tinha entre 18 e 24 anos, estava no curso da primeira gestação, vivia com companheiro, não exercia nenhuma atividade remunerada e tinha mais de 11 anos de estudo.

A distribuição do estado nutricional avaliado pelo nomograma de Rosso, método de Atalah e curva do CLAP das gestantes foi significativamente diferente da distribuição do estado nutricional da população de não gestantes utili- zada para fins comparativos, tanto em nível regional (Nordeste) quanto em nível nacional (Brasil).

Na Tabela 1 acham-se descritos os resultados do estado nutricional das gestantes segundo o nomograma de Rosso e os valores esperados de mulheres não gestantes em idade reprodutiva na Região Nordeste e no Brasil no ano de 2005. As frequências comparativas das classificações em suas diversas categorias (baixo peso, peso normal, sobrepeso/obesidade) divergem substancialmente, principalmente em relação à ocorrência de baixo peso. Houve diferença estatística entre os valores $(p<0,001)$, sendo a condição que

Tabela 1. Comparação entre a classificação do estado nutricional pelo nomograma de Rosso e os valores esperados do estado nutricional de mulheres não gestantes em idade reprodutiva na Região Nordeste-2005 e no Brasil-2005.

\begin{tabular}{|c|c|c|c|c|c|}
\hline \multirow{2}{*}{ Estado nutricional } & \multicolumn{2}{|c|}{ Valores observados } & \multicolumn{2}{|c|}{ Valores esperados para não gestantes da Região Nordeste* } & \multirow{2}{*}{ Contribuição para o $\chi^{2^{* *}}$} \\
\hline & $\mathrm{n}$ & $\%$ & $\mathrm{n}$ & $\%$ & \\
\hline Baixo peso & 392 & 39,5 & 73,4 & 7,4 & 1382,69 \\
\hline Peso normal & 315 & 31,8 & 512,9 & 51,7 & 76,34 \\
\hline Sobrepeso/obesidade & 285 & 28,7 & 405,7 & 40,9 & 35,92 \\
\hline Total & 992 & 100,0 & 992,0 & 100,0 & \\
\hline \multirow{2}{*}{ Estado nutricional } & \multicolumn{2}{|c|}{ Valores observados } & \multicolumn{2}{|c|}{ Valores esperados para não gestantes do Brasil $^{*}$} & Contriburicã n nara \\
\hline & $\mathrm{n}$ & $\%$ & $\mathrm{n}$ & $\%$ & Contriburçao para o X \\
\hline Baixo peso & 392 & 39,5 & 66,5 & 6,7 & 1594,45 \\
\hline Peso normal & 315 & 31,8 & 486,0 & 49,0 & 60,21 \\
\hline Sobrepeso/obesidade & 285 & 28,7 & 439,5 & 44,3 & 54,29 \\
\hline Total & 992 & 100,0 & 992,0 & 100,0 & \\
\hline
\end{tabular}

${ }^{*}$ Fonte: Ministério da Saúde ${ }^{18 ;} ;{ }^{* *} p<0,001$

Tabela 2. Comparação entre a classificação do estado nutricional pela classificação de Atalah e os valores esperados do estado nutricional de mulheres não gestantes em idade reprodutiva na Região Nordeste-2005 e no Brasil-2005.

\begin{tabular}{|c|c|c|c|c|c|}
\hline \multirow{2}{*}{ Estado nutricional } & \multicolumn{2}{|c|}{ Valores observados } & \multicolumn{2}{|c|}{ Valores esperados para não gestantes da Região Nordeste ${ }^{*}$} & \multirow{2}{*}{ Contribuição para o $\chi^{2^{* *}}$} \\
\hline & $n$ & $\%$ & $n$ & $\%$ & \\
\hline Baixo peso & 199 & 20,0 & 73,4 & 7,4 & 214,872 \\
\hline Peso normal & 450 & 45,4 & 512,9 & 51,7 & 7,706 \\
\hline Sobrepeso/obesidade & 343 & 34,6 & 405,7 & 40,9 & 9,698 \\
\hline Total & 992 & 100,0 & 992,0 & 100,0 & \\
\hline \multirow{2}{*}{ Estado nutricional } & \multicolumn{2}{|c|}{ Valores observados } & \multicolumn{2}{|c|}{ 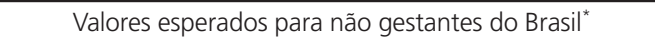 } & \\
\hline & $n$ & $\%$ & $n$ & $\%$ & Contminulça pard O $\chi$ \\
\hline Baixo peso & 199 & 20,0 & 66,5 & 6,7 & 264,290 \\
\hline Peso normal & 450 & 45,4 & 486,0 & 49,0 & 2,678 \\
\hline Sobrepeso/obesidade & 343 & 34,6 & 439,5 & 44,3 & 21,171 \\
\hline Total & 992 & 100,0 & 992,0 & 100,0 & \\
\hline
\end{tabular}

"Fonte: Ministério da Saúde ${ }^{18 ;}{ }^{* *} p<0,001$. 
mais contribuiu para a diferença a de baixo peso $\left(\chi^{2}=1.382,69\right.$ para o Nordeste e $\chi^{2}=1.594,45$ para o Brasil).

A Tabela 2 apresenta a comparação entre os resultados obtidos com a classificação do estado nutricional pelo método de Atalah aplicado às gestantes e os resultados esperados a partir dos valores antropométricos de mulheres não gestantes no Nordeste e no Brasil em 2005. As diferenças extremas foram observadas nas prevalências de baixo peso gravídico por Atalah (20,0\%) e a situação correspondente no grupo de mulheres não grávidas da Região Nordeste $(7,4 \%)$ e do Brasil (6,7\%). No valor do qui-quadrado para cada situação, verificou-se que a menor diferença foi encontrada na categoria de peso normal.

Em relação à curva do CLAP, as maiores diferenças de prevalência entre as gestantes estudadas no IMIP e as mulheres em idade reprodutiva (Região Nordeste) ocorreram na condição de baixo peso: $18,0 \%$ versus $7,4 \%$ para a Região Nordeste e $18,0 \%$ versus $6,7 \%$ para o Brasil em 2005. Já os resultados referentes ao peso normal apresentaram as menores diferenças entre as três categorias comparadas. Para o conjunto das análises estatísticas, o contraste de prevalências referentes ao baixo peso representou a maior parcela de contribuição para o valor final do qui-quadrado (Tabela 3).

\section{I S C U S S Ã O}

A caracterização da amostra, com 92,8\% dos casos provenientes do Recife ou das demais cidades que compõem a Região Metropolitana do Recife, representa, com boa aproximação, o perfil demográfico desse espaço relatado por Batista Filho \& Romani19 ${ }^{19}$ em 2002, na avaliação das condições de saúde materno-infantil do Estado de Pernambuco. Porém, os estudos de base populacional efetuados no Brasil ${ }^{1}$ e no Nordeste ${ }^{20}$ indicam uma evidente reorganização e mudança no perfil do estado nutricional da população adulta, legitimando a base comparativa que se fez em relação aos valores esperados da população de não gestante ${ }^{18}$.

Deve-se observar o fato de não terem sido incluídas gestantes com menos de 18 anos e que a faixa etária de 18 a 24 anos representou 50,7\% da amostra. Esses valores foram compatíveis com a faixa etária encontrada na avaliação da atenção à saúde da criança e da gestante em serviços municipais de saúde, em 2001, em Recife, onde a faixa etária de 18 a 24 anos representou 63,3\% de todo o contingente de mulheres grávidas que demandam os serviços de pré-natal com idade até 24 anos $^{21}$. Tais percentuais traduzem a elevada frequência de gravidez na faixa etária de jovens e adolescentes ${ }^{22,23}$, embora nos últimos anos esses percentuais estejam com tendência decrescente em todo o Brasil24.

Tabela 3. Comparação entre a classificação do estado nutricional de acordo com a curva do CLAP e os valores esperados do estado nutricional de mulheres não gestantes em idade reprodutiva na Região Nordeste-2005 e no Brasil-2005.

\begin{tabular}{|c|c|c|c|c|c|}
\hline \multirow{2}{*}{ Estado nutricional } & \multicolumn{2}{|c|}{ Valores observados } & \multicolumn{2}{|c|}{ Valores esperados para não gestantes da Região Nordeste* } & \multirow{2}{*}{ Contribuição para o $\chi^{2^{* *}}$} \\
\hline & $\mathrm{n}$ & $\%$ & $\mathrm{n}$ & $\%$ & \\
\hline Baixo peso & 179 & 18,0 & 73,4 & 7,4 & 151,866 \\
\hline Peso normal & 464 & 46,8 & 512,9 & 51,7 & 4,656 \\
\hline Sobrepeso/obesidade & 349 & 35,2 & 405,7 & 40,9 & 7,932 \\
\hline Total & 992 & 100,0 & 992,0 & 100,0 & \\
\hline \multirow{2}{*}{ Estado nutricional } & \multicolumn{2}{|c|}{ Valores observados } & \multicolumn{2}{|c|}{ Valores esperados para não gestantes do Brasil ${ }^{*}$} & Contribuicãกn \\
\hline & $\mathrm{n}$ & $\%$ & $\mathrm{n}$ & $\%$ & contribulçao para o $\chi^{2}$ \\
\hline Baixo peso & 179 & 18,0 & 66,5 & 6,7 & 190,545 \\
\hline Peso normal & 464 & 46,8 & 486,0 & 49,0 & 1,003 \\
\hline Sobrepeso/obesidade & 349 & 35,2 & 439,5 & 44,3 & 18,619 \\
\hline Total & 992 & 100,0 & 992,0 & 100,0 & \\
\hline
\end{tabular}

${ }^{*}$ Fonte: Ministério da Saúde ${ }^{18 ;}{ }^{* *} p<0,001$. 
Os métodos de avaliação utilizados neste estudo - a classificação de Rosso ${ }^{14}$, a classificação de Atalah $^{15}$ e a curva do CLAP, proposta de Fescina ${ }^{10}$ - resultaram na detecção de elevadas prevalências (39\%, 20\% e 18\%) de baixo peso nas mulheres avaliadas (proxi da desnutrição materna). Tais valores são muito superiores aos encontrados para as populações de referência utilizadas neste estudo para fins comparativos (7,4\% para a Região Nordeste e de 6,7\% para o Brasil).

O nomograma de Rosso ${ }^{14}$, historicamente o mais usado no Brasil, sempre resultou em elevadas proporções de baixo peso gestacional17,25. Assim, uma análise de 46692 gestantes de serviços de saúde do Estado de Pernambuco no ano 2000, avaliadas pelo nomograma de Rosso, mostrou uma prevalência de $32,0 \%$ de baixo peso gestacional ${ }^{26}$. Outros estudos utilizando essa mesma metodologia resultaram em prevalências bem discrepantes, alcançando $25,7 \%$ na cidade de Viçosa na Bahia ${ }^{7}$ e 56,0\% no Rio de Janeiro9 .

Apesar de a prevalência de baixo peso materno ter sido menor quando avaliada pela classificação de Atalah (20,0\%) e pela curva do CLAP $(18,0 \%)$, esses resultados também não se compatibilizam com os dados utilizados como referência nem tampouco com o quadro epidemiológico da última década na população de mulheres adultas da Região Nordeste, uma vez que a prevalência de baixo peso se situa em torno de $6,0 \%^{27}$. Sob o aspecto epidemiológico, os três métodos supervalorizam um problema praticamente sob controle, pela própria evolução do processo econômico-social (a desnutrição na população adulta), ao mesmo tempo em que subestimam um problema emergente e epidêmico: o sobrepeso/ obesidade ${ }^{28,29}$.

Questiona-se qual dos métodos comparados poderia ser o mais indicado para uso no atendimento rotineiro do pré-natal. O nomograma de Rosso ${ }^{14}$ parece ser o menos indicado. Ainda que a classificação resultante da curva do CLAP possa diminuir as diferenças em relação à ocorrência de desnutrição nas gestantes estudadas, as discrepâncias observadas não permitiriam dizer que ela representaria a "melhor classificação", embora tenha sido a que mais se aproxima da situação encontrada para a população de mulheres não gestantes utilizada aqui para comparativos.

Mesmo considerando que as comparações estejam sendo feitas com mulheres não gestantes, há sem dúvida um grande viés produzido pelos métodos de avaliação antropométrica que são recomendados na gestação. Por que uma população de mulheres não gestantes com prevalências de desnutrição tão baixas teria uma elevação de duas ou três vezes quando se tornam gestantes, ainda que os instrumentos teoricamente considerem e corrijam os acréscimos de peso decorrentes do próprio estado nutricional, incluindo o feto? Essas diferenças persistem mesmo quando se comparam os valores encontrados em inquéritos populacionais realizados no Brasil entre 1974 e 1997, nos quais a prevalência de desnutrição se situou em torno de 15\%, distribuída de forma semelhante entre os dois sexos ${ }^{1}$. Avaliação do estado nutricional em populações de adultos ${ }^{30}$ tem mostrado frequências de baixo peso/desnutrição em torno de 3\% a 4\%. São valores ainda menores que os encontrados na população de mulheres aqui utilizada como referência, em virtude das avaliações terem sido feitas em ambos os sexos. Porém, à medida que o problema da desnutrição em mulheres adultas em idade reprodutiva declinou substancialmente nos últimos anos, outro cenário epidemiológico passou a predominar: a prevalência epidêmica ou mesmo pandêmica do sobrepeso/obesidade no Brasil 1,28,30 e no mundo ${ }^{29}$. No presente estudo, porém, ocorreu o inverso: as prevalências de sobrepeso/obesidade encontradas foram subestimadas em relação aos parâmetros utilizados para comparação, embora com discrepâncias bem menores do que quando comparadas com a frequência do baixo peso. Entre os métodos de avaliação utilizados, o nomograma de Rosso foi o método que mais se distanciou das prevalências esperadas.

As três classificações estudadas, portanto, superestimam as prevalências da desnutrição nas 
gestantes segundo magnitudes que variam de duas a seis vezes, principalmente o nomograma de Rosso. Embora a curva do CLAP tenha sido a que menos se afastou dos resultados da população de mulheres não gestantes utilizada como referência, ela não apresenta os necessários atributos para ser seguramente recomendada como um instrumento satisfatório de avaliação do estado nutricional da gestante. As classificações estudadas, portanto, têm uma utilidade limitada sob o ponto de vista clínico e epidemiológico, na avaliação da condição nutricional na clientela obstétrica.

\section{COLABORADORES}

M.I.B. MELO participou da concepção, da coleta dos dados, da análise, da interpretação dos resultados e da redação do manuscrito. A.I. SOUZA e M. BATISTA FILHO participaram da concepção, da análise e da interpretação dos dados e da redação da versão final do manuscrito. J.N. FIGUEIROA participou da análise e da interpretação dos resultados. J.E. CABRAL-FILHO e M.H.D'A. BENÍCIO participaram da interpretação dos resultados e da redação da versão final do manuscrito.

\section{REFERÊ N CIAS}

1. Acuña K, Cruz T. Avaliação do estado nutricional de adultos e idosos e situação nutricional da população brasileira. Arq Bras Endocrinol Metab. 2004; 48(3):345-61. doi: 10.1590/S0004-273020 04000300004.

2. United Nations Children's Fund. The state of the world's children 2009. Maternal and newborn health. Available from: <http://www.unicef.org/ sowc09/docs/SOWC09-FullReport-EN.pdf>.

3. Batista-Filho $M$, Rissin $A$. A transição nutricional no Brasil: tendências regionais e temporais. Cad Saúde Pública. 2003; 19(Sup. 1):S181-S91. doi: 10.15 90/S0102-311X2003000700019.

4. Atalah SE, Castro SR. Obesidad materna y riesgo reproductivo. Rev Med Chile. 2004; 132(8):923-30. doi: $10.4067 / S 0034-98872004000800003$.

5. Laurenti R, Mello-Jorge MHP, Gotlieb SLD. Reflexões sobre a mensuração da mortalidade materna. Cad Saúde Pública. 2000; 16(1):23-30 doi: 10.1590/\$010 2-311X2000000100003.
6. Nucci LB, Schmidt MI, Ducan BB, Fuchs SC, Fleck ET, Britto MMS. Nutritional status of pregnant women: prevalence and associated pregnancy outcomes. Rev Saúde Pública. 2001; 35(6):502-7. doi: 10.1590/S0034-89102001000600002.

7. Silva EG. Avaliação antropométrica de gestantes: comparação entre três métodos [mestrado]. Salvador: Universidade Federal da Bahia; 2004.

8. Camelo Jr JS. Recém-nascidos de muito baixo peso e estado nutricional: certezas e incertezas. J Pediatr (Rio de Janeiro). 2005; 81(1):5-6. doi: 10.1590/S00 21-75572005000100003.

9. Carmo MGT, Colares IGT, Pereira GS, Soares EA, Sampaio EA, Armada MCS, et al. Avaliação ponderal de gestantes atendidas na Maternidade-Escola da UFRJ e sua relação com o peso ao nascer. J Bras Ginecol. 1998; 108(5):151-7.

10. Fescina RH. Aumento de peso durante el embarazo. Método para su cálculo cuando se desconoce el peso habitual. Bol Oficina Sanit Panam. 1983; 95(2): 156-62.

11. Instituto de Nutrición de Centro-América y Panamá. Evaluación del peso de la embarazada. Guatemala: INCAP; 1961. Nutrición en Salud Pública, NSP, 3.

12. Kac G. Velásquez-Meléndez G. Ganho de peso gestacional e macrossomia em uma coorte de mães e filhos. J Pediatr. 2005, 81(1):47-53. doi: 10.1590/S0 021-75572005000100010.

13. Macéa JR, Kronfly A, Meserani LC. Peso do recémnascido em relação ao ganho ponderal de gestantes normais: análise em diferentes faixas de pesos iniciais maternos. Rev Assoc Med Bras. 1996: 42(3):155-61.

14. Rosso P. A new chart to monitor weight gain during pregnancy. Am J Clin Nutr. 1985; 41(3):644-52.

15. Atalah SE, Castilho CC, Castro RS, Aldea AP. Propuesta de un nuevo estándar de evaluación nutricional en embarazadas. Rev Med Chile. 1997; 125:(12):1429-36.

16. Santander FM. ¿Existe una sola curva de ganância de peso durante el embarazo? RESPYN. 2006 7(2). Disponible: <http://www.respyn.uanl.mx/vii/2/ editorial/editorial-mardones.htm>.

17. Engstron EM. SISVAN: instrumento para o combate aos distúrbios nutricionais em serviços de saúde: o diagnóstico nutricional. Rio de Janeiro: Fiocruz; 2002.

18. Brasil. Ministério da Saúde. Estado nutricional em relação ao CID: adulto [Relatório]. Brasília: MS; 2005.

19. Batista Filho M, Romani SAM. Alimentação, nutrição e saúde no estado de Pernambuco: especialização e fatores socioeconômicos. Recife: IMIP; 2002. Série Publicações Científicas do Instituto Materno Infantil de Pernambuco, n.7. 
20. Monteiro CA, Conde WL. A tendência secular da obesidade segundo estratos sociais: Nordeste e Sudeste do Brasil,1975-1989-1997. Arq Bras Endocrinol Metabol. 1999; 43(3):186-94.

21. Veras AMCA, Osório MM, Frias PG, Sarinho SW, Romani SAM. Avaliação da atenção à saúde da criança e da gestante em serviços municipais de saúde em Recife. Recife: Instituto Materno Infantil de Pernambuco; 2004. Série de Publicações científicas do Instituto Materno Infantil de Pernambuco, n.8.

22. Azevedo GD, Freitas Júnior RAO, Freitas AKMSO, Araújo ACPF, Soares EMM, Maranhão TMO. Efeito da Idade Materna sobre os Resultados Perinatais. Rev Bras Ginecol Obstet. 2002, 24(3):181-5. doi: 10.1590/S0100-72032002000300006.

23. Barbosa AM. Análise sociodemográfica da fecundidade de adolescentes e jovens no Brasil: 1970/2006. Disponivel em: <http://www.abep. nepo.unicamp.br/encontro2008/docspdf/ABEP2 008_1206.pdf>.

24. Brasil. Ministério da Saúde. DATASUS. Indicadores e dados básicos do Brasil: IDB 2007. Brasília: MS; 2008. Disponível em: <http://tabnet.datasus.gov. br/cgi/idb2007/matriz.htm>.

25. Coelho KS, Souza Al, Batista-Filho M. Avaliação antropométrica do estado nutricional da gestante: visão retrospectiva e prospectiva. Rev Bras Saúde
Mater Infant. 2002; 2(1):57-61. doi: 10.1590/\$151 9-38292002000100009.

26. Sistema de Vigilância Alimentar e Nutricional. Dados de serviço de saúde do Estado de Pernambuco. Bol Sisvan. 2000; 11(25):6.

27. Marinho SP, Martins IS, Perestrelo JPP, Oliveira DC. Obesidade em adultos de segmentos pauperizados da sociedade. Rev Nutr. 2003; 16(2):195-201. doi: 10.1590/S1415-52732003000200006.

28. Instituto Brasileiro de Geografia e Estatística. Pesquisa de Orçamentos Familiares. 2002/2003. Disponível em: <http://www.ibge.gov.br/home/ presidencia/noticias/noticia_impressao.php?id_ noticia $=278>$.

29. Scmidhuber J. The growing global obesity problem: some policy options to address it. J Agric Dev Econ. 2004;1(2). Available from: <http://www.fao.org>.

30. Oliveira JS, Lira PIC, Veras ICL, Maia SR, Lemos MCC, Andrade SLLS, et al. Estado nutricional e insegurança alimentar de adolescentes e adultos em duas localidades de baixo índice de desenvolvimento humano. Rev Nutr. 2009; 22(4): 453-65. doi: 10.1590/S1415-52732009000400002.

Recebido em: 26/11/2009

Versão final reapresentada em: 19/7/2010 Aprovado em: 30/8/2010 